\title{
Compatible TDM/WDM PON Using a Single Tunable Optical Filter for Both Downstream Wavelength Selection and Upstream Wavelength Generation
}

\author{
Zhengxuan Li, Lilin Yi, Yan Zhang, Yi Dong, Shilin Xiao, and Weisheng $\mathrm{Hu}$
}

\begin{abstract}
We propose a hybrid time-division multiplexing/ wavelength-division multiplexing passive optical network (TDM/WDM-PON) architecture compatible with the traditional TDM-PON configuration using a power splitter in the remote node. A tunable optical filter (TOF) is used to select the downstream wavelength in each optical network unit. Meanwhile, the TOF plays a second role of seeding a reflective semiconductor optical amplifier (RSOA) so as to construct a tunable fiber-ring laser as colorless upstream source. A stable performance is observed for the upstream laser source tuned from 1535 to $1565 \mathrm{~nm}$ and with a modulation data rate of $1.25 \mathrm{~Gb} / \mathrm{s}$. The upstream wavelength has a $\sim 0.1-\mathrm{nm}$ offset with the central wavelength of the TOF when the RSOA is biased at its saturation region, which mitigates the Rayleigh backscattering and back reflection-induced crosstalk in single-fiber bidirectional PON systems.
\end{abstract}

Index Terms-Reflective semiconductor optical amplifier (RSOA), time-division multiplexing/wavelength-division multiplexing passive optical network (TDM/WDM PON), tunable optical filter (TOF).

\section{INTRODUCTION}

A $S$ the bandwidth demand of each user in access network increases rapidly, the traditional time division multiplexing-passive optical network (TDM-PON) has difficulty in fulfilling the bandwidth requirement. Wavelength division multiplexing-PON (WDM-PON) has been considered as a powerful means to meet the ever-increasing bandwidth demand by introducing multiple wavelengths and using an arrayed waveguide grating (AWG) at remote node (RN) to distribute wavelengths [1]. To increase the number of the users and make full use of the limited wavelength resources, hybrid WDM/TDM PON has attracted a lot of attentions

Manuscript received August 4, 2011; revised January 18, 2012; accepted January 19, 2012. Date of publication February 3, 2012; date of current version April 13, 2012. This work was supported in part by the 973 Program under Grant 2012CB315602 and Grant 2010CB328204-5, in part by the Natural Science Foundation of China under Grant 61007041, Grant 61090393, Grant 61132004, and Grant 60825103, in part by the 863 Program, and in part by the Chen Guang Scholar Program of Shanghai under Program 11CG11.

The authors are with the Department of Electronic Engineering, State Key Laboratory of Advanced Optical Communication Systems and Networks, Shanghai Jiao Tong University, Shanghai 200240, China (e-mail: antlzx@sjtu.edu.cn; lilinyi@sjtu.edu.cn; seeyan@sjtu.edu.cn; yidong@sjtu.edu.cn; slxiao@sjtu.edu.cn; wshu@sjtu.edu.cn).

Color versions of one or more of the figures in this letter are available online at http://ieeexplore.ieee.org.

Digital Object Identifier 10.1109/LPT.2012.2186435 in recent years by combining the features of WDM-PON and TDM-PON together [2,3]. Both WDM-PON and hybrid WDM/TDM-PON configurations require an AWG at RN; therefore it is necessary to update the traditional TDM-PON configuration to support WDM operation. Since it's both costly and troublesome to reconstruct the existing TDM-PON configuration, a new WDM-capable PON structure that has no need of an AWG in the RN is more attractive.

Thisara Jayasinghe $e t$ al. has proposed a multi-wavelength PON (MWPON) without the addition of an AWG in the RN [4], where multi-wavelength downstream signals are broadcasted to all ONUs by a power splitter and wavelength selection is realized by a tunable optical filter (TOF) in each ONU. Centralized laser sources at optical line terminal (OLT) are injected to reflective semiconductor optical amplifier (RSOA) for upstream data modulation. To avoid Rayleigh backscattering and back reflection induced crosstalk in the centralized laser case, a $2 \times \mathrm{n}$ splitter and two sets of feeder fiber are used for downstream and upstream wavelength distribution. Therefore the traditional TDM-PON configuration still needs to be upgraded by replacing the single feeder fiber and $1 \times \mathrm{n}$ splitter with two feeder fibers and a $2 \times \mathrm{n}$ splitter. Besides, two TOFs in each ONU are employed for downstream and upstream wavelength selection respectively, which increases the complexity and cost of each ONU.

In this letter, we propose a novel hybrid TDM/WDM PON architecture to solve the above problems. The downstream wavelength distribution and selection are similar with the MWPON case but using a $1 \times \mathrm{n}$ splitter and a single feeder fiber, which is completely compatible with the traditional TDM-PON configuration. The TOF in each ONU which is used for selecting the downstream wavelength serves a secondary role as seeding a RSOA thereby obtaining a directly modulated tunable fiber ring laser. Therefore only a single TOF is required in each ONU for both downstream wavelength selection and upstream wavelength generation. It's worthy noting that the laser formed in the loop has a wavelength offset of $\sim 0.1 \mathrm{~nm}$ away from the central wavelength of the TOF when the RSOA is biased at its saturation region, which can mitigate the Rayleigh backscattering and back reflection induced crosstalk in the bidirectional single-fiber PON system. Bidirectional transmission of downstream data at $10-\mathrm{Gb} / \mathrm{s}$ and upstream data at $1.25 \mathrm{~Gb} / \mathrm{s}$ per wavelength over $25-\mathrm{km}$ single 


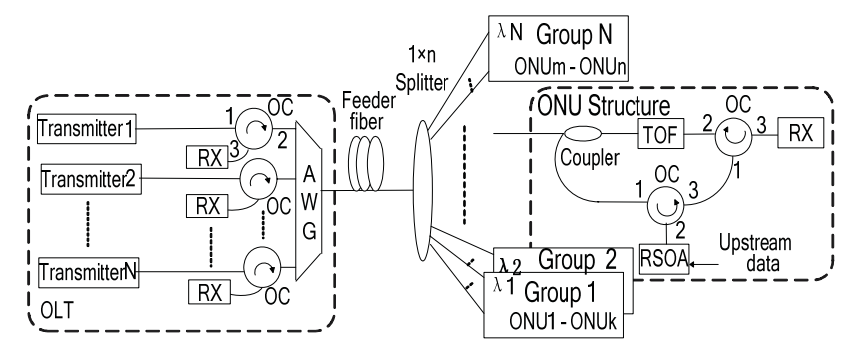

Fig. 1. Proposed hybrid TDM/WDM configuration.

mode fiber (SMF) has been demonstrated with a power penalty less than $2 \mathrm{~dB}$ for both directions when the reflected signal to noise ratio (RSNR) is $18 \mathrm{~dB}$.

\section{HYBRID TDM/WDM-PON ARCHITECTURE}

The configuration of the proposed hybrid WDM/TDM PON is shown in Fig. 1. Downstream wavelengths are multiplexed by an AWG in OLT and distributed into $\mathrm{N}$ groups of ONUs by a $1 \times \mathrm{n}$ splitter in the RN. $\mathrm{N}$ denotes the number of wavelengths and $\mathrm{n}$ represents the number of users. A TOF in each ONU is employed to select the downstream signal by tuning its central wavelength aligned with the desired downstream wavelength. The ONUs in one group share a downstream wavelength in TDM way by tuning the TOFs on the same wavelength, while different ONU groups own different downstream wavelengths in WDM way. The user number in each ONU group is determined by each user's bandwidth demand, which can be dynamically allocated according to the requirements by tuning TOFs.

As for the upstream link, a RSOA based tunable fiber ring laser is used as the upstream laser source. The fiber ring laser consists of a coupler, a TOF, two optical circulators (OCs) and a RSOA. The TOF is also used for downstream wavelength selection. When the RSOA is biased at its saturation region, upstream data can be directly modulated on the RSOA based tunable fiber laser [5]. As TOFs in the same ONU group are tuned on the same wavelength, the upstream lasers in the ONUs also operate at the same wavelength, differing from other ONU groups. With the help of the power splitter, upstream signal from the same group is multiplexed in TDM way, while different groups are multiplexed in WDM way. After passing through the AWG in OLT side, upstream signal from different ONU groups are divided into multi channels and detected by burst mode receivers.

We set up an experiment to investigate the performance of the proposed configuration as shown in Fig. 2. At the OLT side, a tunable laser source (TLS) serves as the downstream laser source. A Mach-Zehnder modulator (MZM) driven by Pseudo-random bit sequence (PRBS) data is used to generate a 10-Gb/s on-off-keying (OOK) downstream data with 13-dB extinction ratio (ER). After 25-km SMF fiber transmission, the signal passes through a coupler, a TOF and OC2, finally detected by an optical receiver. The central wavelength of the TOF is aligned to the downstream wavelength for minimizing

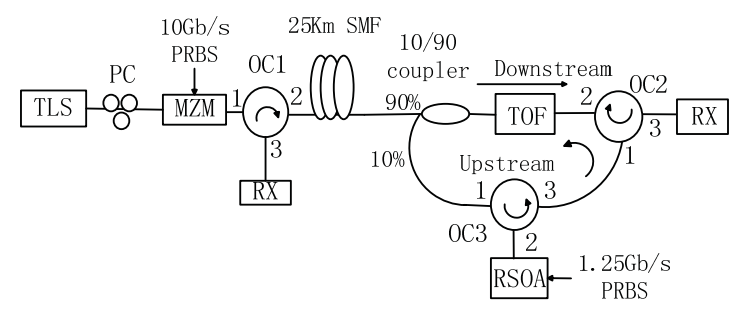

Fig. 2. Experimental setup.

the downstream loss. For a higher power budget for both downstream and upstream reception, a 10/90 coupler is used. The coupler launches $90 \%$ output power of the fiber laser into the transmission fiber as the upstream source. By tuning the bias current of the RSOA at its saturation region, the RSOA based tunable fiber ring laser can be directly modulated with a data rate up to $2.2 \mathrm{~Gb} / \mathrm{s}$, which is limited by the RSOA electrical bandwidth. In the experiment, we use $1.25 \mathrm{~Gb} / \mathrm{s}$ upstream data rate to meet the GPON requirement. Higher modulation data rate such as $10 \mathrm{~Gb} / \mathrm{s}$ could be achieved by employing forward error correction (FEC) and electrical equalization techniques at OLT.

\section{EXPERIMENTAL RESUlTS AND Discussion}

We first investigated the performance of the proposed fiber ring laser as the upstream colorless source. The TOF has a 3 -dB bandwidth of $\sim 0.4 \mathrm{~nm}$ and a laser is formed in the fiber ring with the help of TOF. By increasing the bias current, the RSOA gain linearly increases firstly and then achieves saturation at its high gain region. We discovered that the output wavelength of the fiber ring laser shifts accordingly with the tuning of the bias current as shown in inset (a) of Fig. 3, and a $\sim 0.1-\mathrm{nm}$ offset is observed at its saturation region, the exact region where upstream data can be directly modulated on the RSOA. The frequency red-shift is attributed to self-phase modulation (SPM) induced frequency chirp in the saturated RSOA, which has been observed in saturated SOA [6]. The clear upstream eye diagram with $1.25-\mathrm{Gb} / \mathrm{s}$ data rate is shown in inset (b) of Fig. 3, with an ER of around $8 \mathrm{~dB}$. We only take $1535 \mathrm{~nm}$ and $1550 \mathrm{~nm}$ as examples in Fig. 3, but the measured performance is the same for wavelengths tuning from $1535 \mathrm{~nm}$ to $1565 \mathrm{~nm}$. Fig. 4 shows the optical spectra of the upstream tunable fiber laser from $1535 \mathrm{~nm}$ to $1565 \mathrm{~nm}$ with a measuring step of $5 \mathrm{~nm}$. Within the entire range, the laser features side mode suppression ratio (SMSR) higher than $60 \mathrm{~dB}$, output power higher than $-1.5 \mathrm{dBm}$ and power ripple less than $1 \mathrm{~dB}$, which is a perfect upstream colorless source.

We then investigated the proposed hybrid TDM/WDMPON configuration by testing the transmission property. To investigate the flexibility of the fiber ring laser within the whole bandwidth of the RSOA, we measured the bit error rate (BER) and eye diagrams of upstream signals at $1535 \mathrm{~nm}$ and $1550 \mathrm{~nm}$ as shown in Fig. 5 (a). The sensitivities are similar and almost no power penalty is observed after transmission, verifying a stable performance of the upstream laser source on different operation wavelengths. 


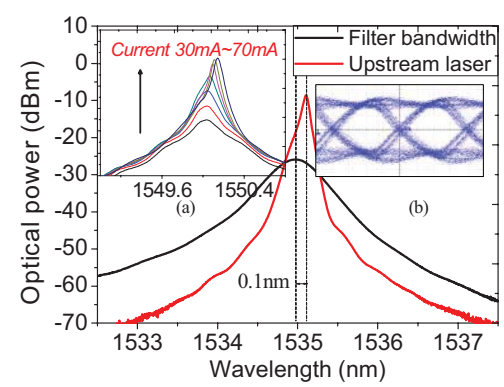

Fig. 3. Wavelength offset of the upstream laser.

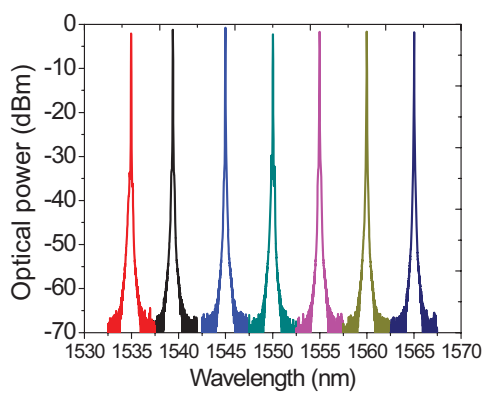

Fig. 4. Optical spectra of the laser output.

Taking into account the total loss in the system of $\sim 10.5 \mathrm{~dB}$, including an AWG, the $25-\mathrm{km} \mathrm{SMF}$ and an OC, and the worst sensitivity of the upstream signal of $\sim-29 \mathrm{dBm}$, the power budget is $\sim 17 \mathrm{~dB}$, therefore the proposed PON could support a 1:32 splitting ratio with a $2-\mathrm{dB}$ power margin. By using a RSOA with higher linear gain, the power budget can be improved. Moreover, to verify the shift between upstream and downstream wavelengths can mitigate the back-reflection and Rayleigh backscattering induced crosstalk, we set the RSNR at $18 \mathrm{~dB}$ by intentionally introducing reflection at the fiber end and measured the BER of the downstream and upstream signals at $1550 \mathrm{~nm}$ in back-to-back (BtB) case, after $25-\mathrm{km}$ SMF bidirectional transmission with and without a $0.1-\mathrm{nm}$ wavelength shift, as shown in Fig. 5(b). The sensitivities of signal in both directions are measured by using an erbium-doped fiber amplifier (EDFA) as a pre-amplifier since a PIN rather than an APD is used for signal detection in the experiment. The EDFA is not necessary if an APD is employed for higher sensitivity. It turns out that without the wavelength shift, both upstream and downstream signals are severely distorted and the error-free transmission cannot be achieved, but with the $0.1-\mathrm{nm}$ shift, the eye diagrams are clearly open again and the error-free transmission is achieved with a power penalty less than $2 \mathrm{~dB}$ in both directions, proving that the offset between downstream and upstream wavelength can effectively mitigate the back-reflection and Rayleigh backscattering induced crosstalk. Besides, the Rayleigh backscattering of the upstream signal cannot enter the RSOA for re-amplification due to the use of the circulator; therefore Rayleigh backscattering crosstalk induced by the upstream signal itself is also avoided. All the results above demonstrate the feasibility of using a same TOF for downstream wavelength selection and upstream wavelength generation in the proposed hybrid TDM/WDMPON configuration.
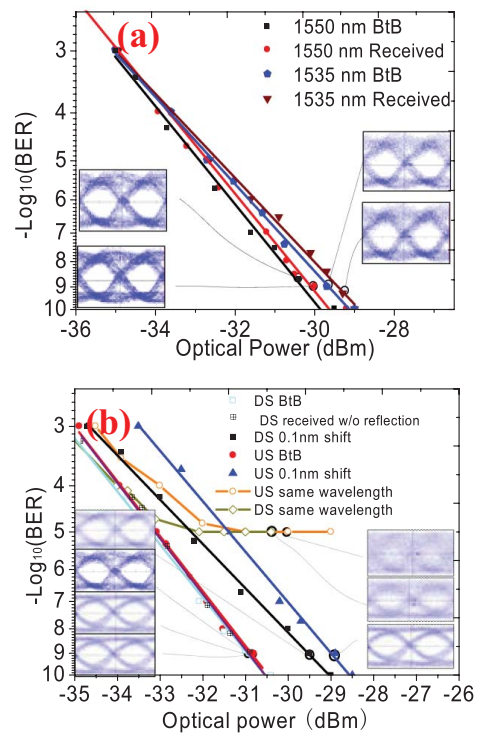

Fig. 5. (a) BER and eye diagrams measurement of the upstream signals. (b) Rayleigh backscattering and back-reflection mitigation measurement.

\section{CONCLUSION}

We have proposed a hybrid TDM/WDM-PON configuration based on the traditional TDM-PON structure using a directly modulated tunable fiber ring laser based on RSOA as the colorless upstream source. Downstream wavelength selection is achieved with the help of a TOF in each ONU, enabling a hybrid TDM/WDM-PON compatible with the traditional TDM-PON configuration. Meanwhile, the TOF serves a secondary role as seeding the RSOA for upstream laser generation. The transmission performances of the upstream laser source are experimentally investigated and it turned out that the offset between upstream and downstream wavelength can effectively mitigate the back reflection and Rayleigh backscattering induced crosstalk. Transmission capacity of the hybrid TDM/WDM PON can be upgraded by introducing more wavelengths at OLT and tuning the operating wavelength of the ONU to another one differing from others according to the user's demand.

\section{REFERENCES}

[1] A. Banerjee, et al., "Wavelength division-multiplexed passive optical network (WDM-PON) technologies for broadband access: A review," $J$. Opt. Netw., vol. 4, no. 11, pp. 737-758, Nov. 2005.

[2] C. Bock, J. Prat, and S. D. Walker, "Hybrid WDM/TDM PON using the AWG FSR and featuring centralized light generation and DBA," $J$. Lightw. Technol., vol. 23, no. 12, pp. 3981-3988, Dec. 2005.

[3] C.-J. Chae and T. Jayasinghe, "bandwidth-efficient capacity upgrade of ethernet passive optical network systems," Electron. Lett., vol. 42, no. 16, pp. 938-940, Aug. 2006.

[4] T. Jayasinghe, C. J. Chae, and R. S. Tucker, "Scalability of RSOA-based multiwavelength Ethernet PON architecture with dual feeder fiber," $J$. Opt. Netw., vol. 6, no. 8, pp. 1025-1040, Aug. 2007.

[5] Z.-R. Lin, C.-K. Liu, Y.-J. Jhang, and G. Keiser, "Tunable directly modulated fiber ring laser using a reflective semiconductor optical amplifier for WDM access networks," Opt. Express, vol. 18, no. 17, pp. 17610-17619, Aug. 2010.

[6] G. P. Agrawal and N. A. Olsson, "Self-phase modulation and spectral broadening of optical pulses in semiconductor laser amplifiers," IEEE J. Quantum Electron., vol. 25, no. 11, pp. 2297-2306, Nov. 1989. 